\title{
Pyogenic Liver Abscess after Bile Duct Exploration Drained with Endoscopic Techniques
}

\section{Jun Lu, Yan-Hui Zhu, Zhi-Tian Li and Zhong Jia*}

Department of Hepatopancreatobiliary Surgery, Hangzhou First People's Hospital (Nanjing Medical University Affiliated Hangzhou Hospital), China

\begin{abstract}
Background: Pyogenic liver abscess (PLA) after surgery may lead to sepsis.Endoscopic techniques provide an alternative choice for PLA drainage.

Methods: We describe a 49-year-old woman who developed a right PLA after left lobe hepatectomy and common bile duct (CBD) exploration due to intra-and extra-hepatic bile duct stones.

Results: The patient suffered from persistent high fever and hyperbilirubinemia with unknown reason after surgery despite amount of antibiotic applied until a liver abscess in right lobe was found .It was treated with ultrasound-guided percutaneous transhepatic drainage(PTD). However, outcome of the PTD was worse because of no fluid collected in abscess cavity at that time. T-tube drainage suddenly stop work after post-operative day 4. Emergent Endoscopic Retrograde Cholangiopancreatography (ERCP) and Endoscopic Nasobiliary Drainage (ENBD) was performed. The patient recovered rapidly a week later.
\end{abstract}

Conclusions: PLA induced by regional closed-loop biliary obstruction is an important reason of abdominal infection after surgery.Endoscopic techniques with ERCP and ENBD can resolve PLA in early stage especially when PTD inadequently drained.

Keywords: Endoscopic nasobiliary drainage; Endoscopic retrograde cholangiopancreatography; Drainage; Pyogenic liver abscess

\section{Introduction}

A PLA is a "pocket" of pus that forms in the liver in response to bacterial infection, amoebic infection. Trauma or a range of unknown cryptogenic causes. The morbid PLA patients more likely prone to suffer higher risk of development of sepsis or multiple organ failure due to greater insulin, diabetes, malignancy, antibiotic resistance, inadequate abscess drainage, and some surgeries such as bile duct exploration unless they get proper management [1]. PLAs in control is a stateof-the-art. Majority of PLAs could be drained by minimally invasive therapy including ultrasound-guided PTD [2], the others have to face surgical therapy $[3,4]$. However, PLAs after surgery is seldom reported, which will push surgeons into a "dilemma" situation when noninvasive therapy always failures to drain immature PLAs. Herein, we would like to present this case report to share our therapeutic experiences by endoscopic techniques for PLAs after surgery.

\section{Case Report}

A 49-year-old woman with a 2-year history of recurrent episodes of upper abdominal pain often combined with hyperbilirubinemia and high fever, was admitted to hospital. This time, she was diagnosed with it and recommended surgery. Imaging examinations revealed dilated left hepatic lobe ducts with multiple filling defects consistent with stones combined with right hepatic duct stricture and mild dilatation. The patient was performed laparoscopic left hepatic resection and hepaticolithotomy with T-tube drainage. After the post-operative day 4, the volume of drainage decreased around $15 \mathrm{ml}$ and the fluid was turbid. The patient occurred high fever, right quadrant abdominal dull-like pain. Her laboratory data demonstrated leukocytosis, hyperbilirubinemia, the peak volume of total bilirubin of $113 \mu \mathrm{mol} / \mathrm{L}$, the peak volume of conjugated bilirubin of $73.1 \mu \mathrm{mol} / \mathrm{L}$, persistent $\mathrm{C}$-reactin protein of more than $160 \mathrm{mg} / \mathrm{L}$.

It appears to be not easy to identify the definite cause of fever or address the primary focus of infection. Bile culture and blood culture suggested multi-drug resistant bacteria including E. coli, Pseudomonas aeruginosa, and Staphylococcus. The antibacterial therapy including
Tigecycline seemed to have no effects at all. Re-CT scan found a large liver abscess existing in right lobe just above right hepatic duct stricture. As the less liquid formed in the focus of liver abscess, a percutaneous transhepatic catheter was placed into the right lobe liver abscess, but such intervention was repeatedly unsuccessful at draining the abscess. In addition, food debris and turbid fluid can be drained from T-tube.

Then the further CT scan revealed the abscess was larger than before and intra-hepatic bile duct was found ruptured with bile flowing into parenchyma of liver abscess (Figure 1A). The clinical presentation of the patient had been persistent for about one half a month, so urgent ERCP with the nasobiliary duct placement through stricture of the right hepatic duct to fully drainage (Figure $2 \mathrm{~A}$ and $2 \mathrm{~B}$ ) had to be applied temporary finally. All symptoms alleviated gradually and recovered completely in the end (Figure 1B).

\section{Discussion}

PLA is an uncommon disease, with $8 \sim 20$ per 100,000 hospital admissions [5]. In last 10 decades, many interventional techniques such as ultrasound-guided PTD were applied increasingly [6]. However, PLA is not easy to drain successfully with PTD when only a little pus accumulated in the abscess cavity. Sometimes the liver abscess keeps an immature stage even if amount of antibiotics are applied [6]. The patient will be pushed into a deadly risk of sepsis.

*Corresponding author: Zhong Jia, Department of Hepatopancreatobiliary Surgery, Hangzhou First People's Hospital, Nanjing Medical University, Huansha Road 261, Hangzhou, China, Tel: +86-13958114181; Fax:+86-0571-87914773 E-mail: jiazhong20058@hotmail.com

Received August 16, 2016; Accepted August 31, 2016; Published September 08, 2016

Citation: Lu J, Zhu YH, Li ZT, Jia Z (2016) Pyogenic Liver Abscess after Bile Duct Exploration Drained with Endoscopic Techniques. Surgery Curr Res 6: 277. doi:10.4172/2161-1076.1000277

Copyright: (c) $2016 \mathrm{Lu} \mathrm{J}$, et al. This is an open-access article distributed under the terms of the Creative Commons Attribution License, which permits unrestricted use, distribution, and reproduction in any medium, provided the original author and source are credited. 
Citation: Lu J, Zhu YH, Li ZT, Jia Z (2016) Pyogenic Liver Abscess after Bile Duct Exploration Drained with Endoscopic Techniques. Surgery Curr Res 6: 277. doi:10.4172/2161-1076.1000277
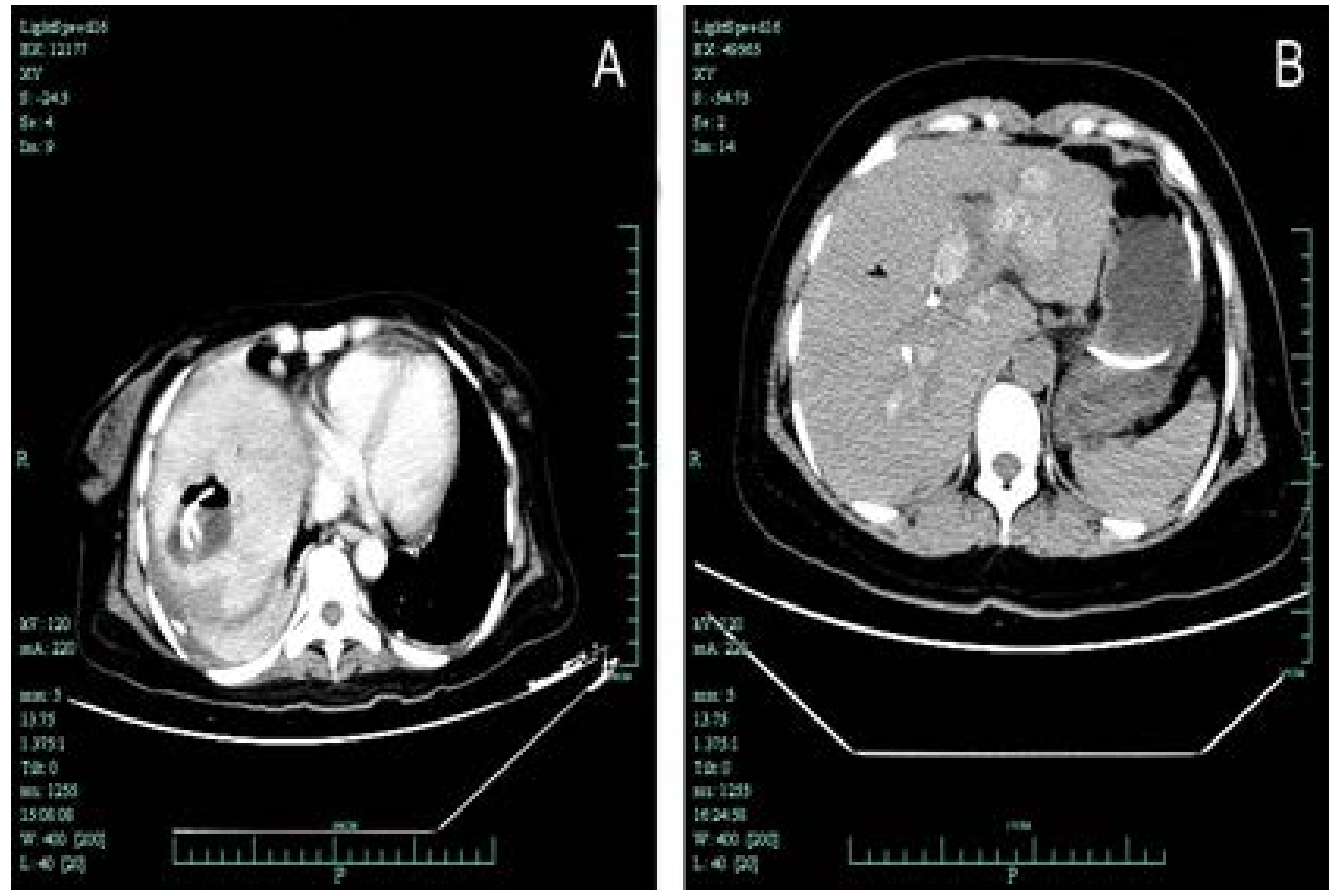

Figure 1: A) CT scan of the abdomen showing a hepatic abscess in the right lobe, in communication with ruptured intrahepatic bile duct, both of which contain gas. The cholestasis exists in the proximal to the biliary stricture; B) CT scan of the abdomen showing the hepatic abscess in the right lobe reduced gradually post-operative day 60 after the procedure of ERCP.
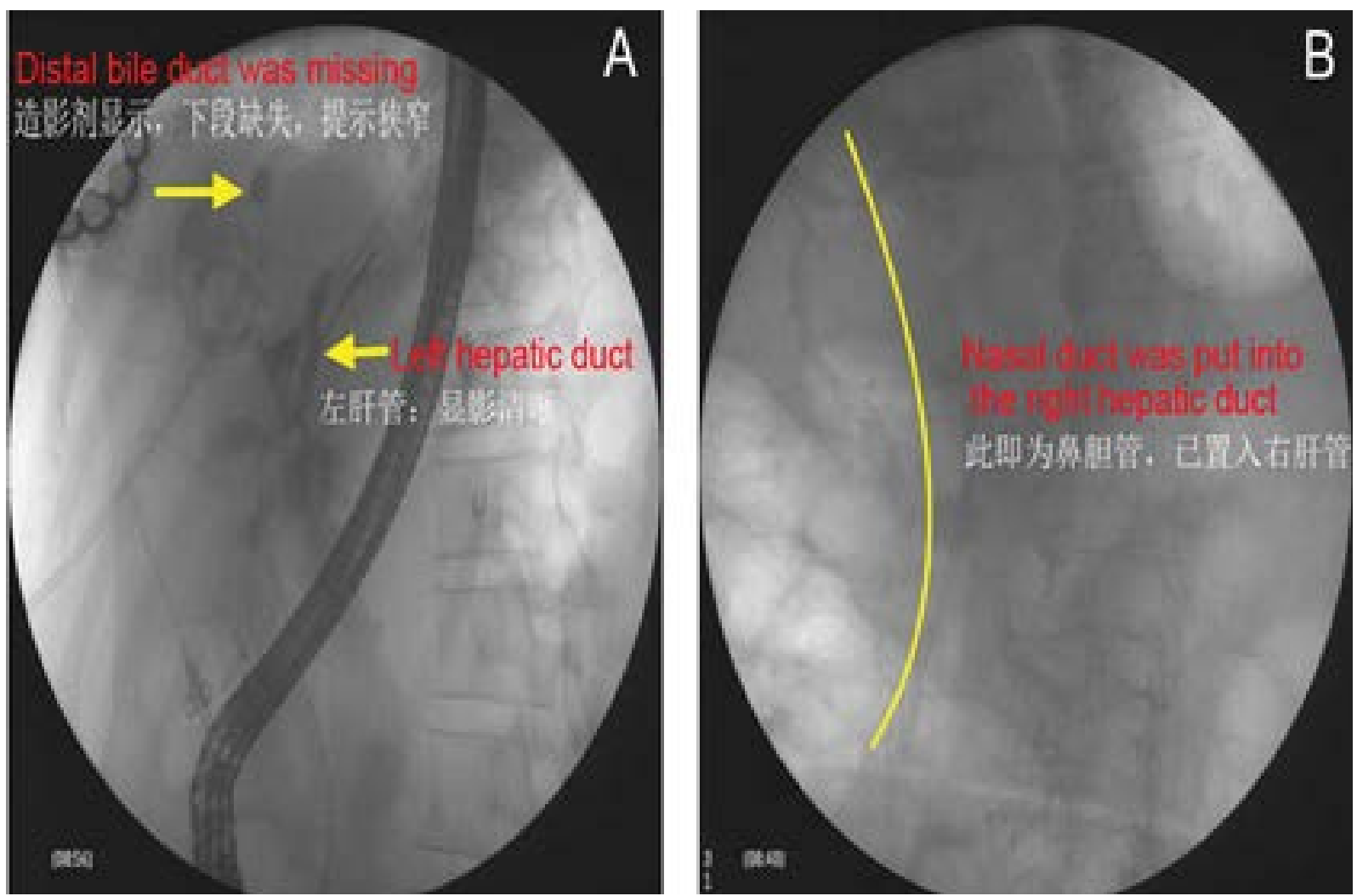

Figure 2: A) Endoscopic retrograde cholangio-pancreatography showing a stricture of the right hepatic duct; B) ERCP showing placement of nasobiliary duct into the abscess zone and pyogenic bile was drained successfully. 
The infection spreads to the liver via various routes, of which the most identifiable cause is biliary-related [7]. Most of microbes are multi-drug resistant, which led to the liver abscess reduced slowly. As a result, effective drainage become more important compared to antibiotics therapy. Based on this case, the placement of nasobiliary duct via II grade bile duct is feasible and accessible.

The definite microbiology is obtained either by puncture biopsy or various samples related to abscess (bile, blood and drainage from abscess). The bacteria culture and the bacteria sensitivity test allow the appropriate choice of antibiotics. The duration of antibiotic should persist for $2 \sim 6$ weeks [8]. Surgical drainage is not essential for most of the patients with PLA, but required, especially when the abscess is larger than $3 \sim 5 \mathrm{~cm}$ in diameter and the antibiotic therapy fails [9]. The post-operative PLA is quite rare, only sparkly reported [10]. Therefore, PLA after surgery common bile duct (CBD) exploration or other biliary tree involved surgery should be suspected when unknown fever remains for a long time. Based on our experiences, there are three clinical characters may do great help to early diagnosis. The volume of T-tube drainage declines sharply $\mathrm{B}$. Continuous high fever $\left(\mathrm{T}>3^{\circ} \mathrm{C}\right)$. New emerging focus of liver found in imaging examinations.

There are many factors leading to the post-operative PLA formation. Most of them contributed to ischemia cholangitis and/ or biliary stricture [11]. Herein, T-tube drainage decreases suddenly from approximately $200 \mathrm{ml}$ to $15 \mathrm{ml}$ and subsequently the food residue refluxes into $\mathrm{CBD}$. The phenomenon may be explained by balance of pressure broken between biliary tract and intestinal tract. The right hepatic stricture and its upstream cholestasis co-exist will result in local close-loop bile duct obstruction, which introduces enteric reflux and bacterial contamination.

Theoretically, mini-invasive procedures including final needle aspiration and persistent catheter drainage will be performed successfully for most of PLA in the nature status [12]. However, the drainage often fails in early stage of PLA because little free fluid found in imaging graphy. ERCP is widely used to diagnose and treat biliary disease, but has never been reported before for PLA. In this case, nasobiliary duct was placed into the parenchyma of abscess through the biliary stricture, the pus in the abscess cavity was drained promptly. The patient recovered 2 weeks later and all symptoms disappeared.

Multiple discipline team for PLA (interventional radiologist, infection experts, surgeon and digestive physician) is of crucial importance, which will help us to set up a proximal protocal or adjust it timely according to the development of PLA. In our practice, antibiotic should be used as a basic step to control infection from begining of PLA until the size of PLA reduced $(<2 \mathrm{~cm})$ and $C$ reactin protein is normal. Antibiotic should be choosed according to the result of antibiotic sensitive test rather than our previous clinical experience. If the PLA becomes mature, repeatedly PTD may be effective. As a result, most of PLAs reduce in size gradually. Otherwise, fifteen percent of patients with PLA may lead to death. As we all well know nearly $90 \%$ of infection spread into liver via bile route or biliary tree-related factors (bile duct injury, abnormal anatomy of biliary tree, bile duct ischemia, endoscopic procedure, et al). So, it may be accessible to drain PLA in its early stage (immature or premature abscess) if PLA locates nearly major biliary tree. On second look, in this case, the endoscopic drainage earlier, the outcome better. Sometimes, it is difficult to distinguish from other diseases (inflammatory mass, tumor), which will challenge the surgeon's decision-making. When all nonsurgical therapies are ineffective like expected, or PLA with a thickening wall, it's time for surgery.

\section{Conclusion}

ERCP and ENBD may be an alternative choice in the future for PLA drainage. The team-work for PLA allows the promise for better outcome.

\section{References}

1. Alvarez Pérez JA, González JJ, Baldonedo RF, Sanz L, Carreño G, et al (2001) Clinical course, treatment, and multivariate analysis of risk factors for pyogenic liver abscess. Am J Surg 181: 177-186.

2. VogI TJ, Estifan F (2001) [Pyogenic liver abscess: interventional versus surgica therapy: technique, results and indications]. Rofo 173: 663-667.

3. Ng SS, Lee JF, Lai PB (2008) Role and outcome of conventional surgery in the treatment of pyogenic liver abscess in the modern era of minimally invasive therapy. World J Gastroenterol 14: 747-751.

4. Tu JF, Huang XF, Hu RY, You HY, Zheng XF, et al. (2011) Comparison of laparoscopic and open surgery for pyogenic liver abscess with biliary pathology. World J Gastroenterol 17: 4339-4343.

5. Chen SC, Tsai SJ, Chen CH, Huang CC, Lin DB, et al. (2008) Predictors of mortality in patients with pyogenic liver abscess. Neth J Med 66: 196-203.

6. Jamil B, Hamid SS (2002) Abscesses in the liver: amoebic or pyogenic? J Pak Med Assoc 52: 495-496.

7. Meddings L, Myers RP, Hubbard J, Shaheen AA, Laupland KB, et al. (2010) A population-based study of pyogenic liver abscesses in the United States: incidence, mortality, and temporal trends. Am J Gastroenterol 105: 117-124.

8. Liu Y, Wang JY, Jiang W (2013) An Increasing Prominent Disease of Klebsiella pneumoniae Liver Abscess: Etiology, Diagnosis, and Treatment.Gastroenterol Res Pract.

9. Njoku VC, Howard TJ, Shen C, Zyromski NJ, Schmidt CM, et al. (2014) Pyogenic liver abscess following pancreaticoduodenectomy: risk factors treatment, and long-term outcome. J Gastrointest Surg 18: 922-928.

10. Matthews JB, Gertsch P, Baer HU, Blumgart LH (1990) Hepatic abscess after biliary tract procedures. Surg Gynecol Obstet. 170: 469-475.

11. Lardière-Deguelte $S$, Ragot $E$, Amroun K, Piardi T, Dokmak S, et al. (2015) Hepatic abscess: Diagnosis and management. J Visc Surg 152: 231-243.

12. Cai YL, Xiong XZ, Lu J, Cheng Y, Yang C, et al. (2015) Percutaneous needle aspiration versus catheter drainage in the management of liver abscess: a systematic review and meta-analysis.HPB (Oxford) 17: 195-201. 